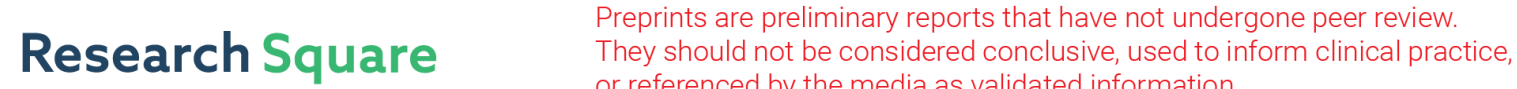 or referenced by the media as validated information. \\ Diet quality and its relationship with overweight and laboratory parameters in breast cancer patients undergoing treatment
}

Krystal Ng LS

Universiti Putra Malaysia

\section{Zalilah MS}

Universiti Putra Malaysia

Chan Yoke Mun ( $\nabla$ yokemun_chan@yahoo.com )

Universiti Putra Malaysia https://orcid.org/0000-0002-3853-736X

Research article

Keywords: Breast cancer, Diet quality, Overweight, Serum hemoglobin

Posted Date: February 17th, 2020

DOI: https://doi.org/10.21203/rs.2.11380/v4

License: (1) (i) This work is licensed under a Creative Commons Attribution 4.0 International License.

Read Full License 


\section{Abstract}

Background Overweight is the common issue existed among breast cancer patients, leading to poor cancer prognosis and survival. However, previous studies have focused merely on single nutrients or food groups. This study aimed to determine whether diet quality was associated with overweight and laboratory parameters among breast cancer patients undergoing treatment. Methods A cross sectional study comprises of 163 patients was conducted from January to May 2017. Diet quality and level of physical activity of patients were ascertained using Healthy Eating Index (HEI)-2015 and International Physical Activity Questionnaire (IPAQ)-short form, respectively. Data were available on body mass index and biochemical data, including of serum albumin, serum hemoblogbin and neutrophils count. Logistic regression was performed to identify the association between diet quality and overweight, serum albumin, serum hemoglobin and neutrophils count. Results The mean total HEl score (standard deviation) of the breast cancer patients was 63.86 (8.75). More than $80 \%$ of the subjects did not adhere to the dietary guidelines for whole grains, dairy, refined grains, ratio of poly- and mono-unsaturated fatty acids to saturated fatty acids components. In the multivariate model, those who perceived higher score of total HEl-2015 were less likely to experience overweight (Q4 vs Q1: OR $=0.36 ; 95 \% \mathrm{Cl}=0.13,0.99$ ) while reported higher concentration of serum hemoglobin (Q4 vs Q1: OR=0.36; $95 \% \mathrm{Cl}=0.13,0.98$; Q3 vs Q1: $\mathrm{OR}=0.35 ; 95 \% \mathrm{Cl}=0.13,0.92$ ). Non-significant associations of diet quality were shown for serum albumin and neutrophils count. Conclusions A majority of breast cancer patients require dietary modification, especially for fatty acids, grains and dairy products. Diet quality may associate with overweight. A balanced diet that address the problem of overweight may help to maintain a good nutritional status and to improve cancer care in breast cancer patients during treatment. Future prospective studies are required to confirm this association.

\section{Background}

Cancer- and treatment-related factors have resulted in various undesirable health outcomes among the cancer patients. Unlike other cancers such as head and neck, gastrointestinal tract, liver and lung cancers, patients with breast cancer have lower risk of undernutrition ${ }^{1,2}$. Weight gain is the common issue among breast cancer patients undergoing treatment ${ }^{3}$, which may have detrimental effect on survival as a result of inflammation ${ }^{4,5}$. In addition, breast cancer patients often suffer from anemia and neutropenia ${ }^{6}$, leads to poor prognosis. While low serum hemoglobin is related to increased level of fatigue, reduced ability to perform daily tasks and impaired quality of life ${ }^{6,7}$, an abnormally low neutrophils count predisposes cancer patients to bacterial infection, which could be life-threatening ${ }^{8}$. Such negative impact of altered blood readings on patients' prognosis might indirectly influence treatment compliance, in light of the close linkage between quality of life and treatment compliance ${ }^{9}$.

In addition to tobacco use, physical activity and diet are established modifiable factor of body weight ${ }^{10}$ via principle of energy imbalance. A prospective study highlighted that breast cancer patients had low self-reported physical activity level especially during treatment ${ }^{11}$, which could be in relation to the 
treatment-induced fatigue or lymphedema. In regard to dietary assessment, diet quality measure provides a better insight on the overall picture of diet consumed in accordance with dietary guidelines, rather than depend on single nutrient ${ }^{12}$. This tool has been extensively used for epidemiological studies, in regard to the risk prediction of various health outcomes ${ }^{12}$. In addition to the reduction in cancer risk ${ }^{13}$, healthier diet was found to associate with reduced inflammation ${ }^{14}$ and improved prognosis among breast cancer survivors ${ }^{15,16}$. The health benefit of diet could be explained by the food synergy concept ${ }^{17}$, in which each of the nutrients acts simultaneously to yield an effective biological pathway. Conversely, Wu (2016) highlighted that insufficient protein intake could solely compromise the immune function ${ }^{18}$, which may forsake such notion that an evaluation of combined food is more informative in regards to a particular health issue. Nevertheless, how diet quality may associate with overweight and altered biochemical parameters for breast cancer patients, at the critical phase of on-going treatment is relatively sparse.

As poor diet is commonly perceived to be linked with cancer developement ${ }^{19,20}$, cancer patients are likely to consume low fat, high fiber diet upon diagnosis ${ }^{21,22}$. Nonetheless, few studies highlighted the reduced diet quality during treatment among breast cancer patients, which resulted from the poor adherence to dietary guidelines for the components of fruits and vegetables ${ }^{23,24}$, grains, solid fats, alcohol and added sugars $^{23}$. This could be explained by the altered taste or increased eating pleasure for "heavy taste" food during treatment ${ }^{25}$. Preference for spicy and salty food was found to associate with overweight in breast cancer patients, in light of its potential impact on increased overall appetite via oral sensory stimulation ${ }^{25}$. Diet quality covers the evaluation of a combination of food groups and nutrients from the whole food consumed, whereby the diet-disease relationship could be more detectable ${ }^{12}$. This is very beneficial for the successful implementation of dietary strategies. The dietary guidelines help to promote healthy eating via the key principles of balanced, moderation, adequacy and variety, and so to maintain a healthy weight during cancer treatment.

To the best of our knowledge, this is the first study investigating the relationships between overall diet consumed and overweight and altered laboratory parameters in breast cancer patients undergoing treatment. This study included only non-terminally ill breast cancer patients, who were susceptible to poor dietary habit as a result of changes in taste perception while receiving treatment ${ }^{25}$. Evidence is limited on which particular dietary patterns might influence clinical outcomes such as recurrence and mortality among cancer survivors ${ }^{26}$. In light of the possibilities of epigenetic aberrations occurring in cancer which could be altered by nutrients makes it plausible that dietary changes after successful cancer treatment could improve prognosis ${ }^{27}$, identification of dietary pattern of non-terminally ill breast cancer patients is needed. A balanced diet could be an impetus for alleviated side effects which resulting from cancer or its treatment. The findings may assist the dieticians to identify patients who need improvement in their dietary intake, thus integrating nutritional care into quality cancer care. However, little is known about the linkage of diet quality with overweight and the common treatment-induced abnormal laboratory values. This study sought to determine the diet quality of breast cancer patients at non-terminal stage as well as 
its association with overweight and treatment-related side effects of hypoalbuminemia, low serum hemoglobin and low neutrophils count.

\section{Methods}

\section{Design}

This was a cross sectional study.

\section{Samples}

This study was conducted among breast cancer patients in National Cancer Institute, which is located in Federal Territory of Putrajaya. There were approximately 20 new cases of breast cancer reported each month in National Cancer Institute. Given that number of predictors $=11$, the minimum sample size required based on the formula of Green (1919), $50+8 k$ was 138 . The eligible patients were enrolled using purposive sampling. The inclusion criteria were female, aged $29-71$ years, receiving treatments $(\geq 1$ course) and able to provide written consent. Patients were excluded if under hospice care or have life expectancy of $\leq 6$ months, pregnant or lactating, diagnosed with severe mental disorders or reduced ability to understand or communicate with others.

\section{Data collection}

The data collection took five months (January - May 2017) and was carried out on patients' appointment day of treatment administration. Ethics approval of this study protocol was obtained from the Ministry of Health's Research and Ethics Committee (MREC) and Ethic Committee for Research Involving Human Subject (JKEUPM), Universiti Putra Malaysia, with written informed consents obtained from all patients prior to study enrolment.

\section{Measurements}

Measurements of weight and height were taken to the nearest $0.1 \mathrm{~kg}$ and $0.1 \mathrm{~cm}$, respectively, by using Detecto bariatric scale (6857DHR). Overweight was defined according to body mass index (BMI) cut off point of $\geq 25.00 \mathrm{~kg} / \mathrm{m}^{2} 28$.

Habitual dietary intake of patients over the past one month was assessed using a 165 -items semiquantitative Malaysians' Food Frequency Questionnaires (FFQ) ${ }^{29}$. Dietary data were analysed using Nutritionist Pro version 4.0.0 (Axxya system, 2017) based on Malaysian food composition database as the primary source, and supplemented by Singaporean and USDA database (if there's lacking of food items in Malaysian database). Food consumption data was transformed into Healthy eating index-2015 (HEl-2015) scoring according to nine adequacy and four moderation components ${ }^{30}$. Each food component was scored proportionately between the minimum and maximum standard as shown in Table 1. The summation of individual components yields a total HEI-2015 score, with higher score indicates better adherence to Dietary Guidelines for Americans 2015-202030. The total HEI-2015 score was divided 
into quartiles. In view of the lack of well-validated diet quality tool for Malaysians ${ }^{31}$, the decision of utilizing HEl-2015 tool was made to assess patients' diet quality as it covers different food groups or subgroups which enables the result to be more discussable.

International Physical Activity Questionnaire (IPAQ)-short form was used to assess level of physical activity level of patients. Total intensity level of physical activity (MET-min/week) was identified by taking consideration into frequency and duration of each activity type at one time. The higher reading of METs score indicates more intense physical activity. Patients' physical activity level was categorized into low and moderate or high according to the total frequency, duration and intensity level of physical activities that have been carried out over the past one week ${ }^{32}$.

Demographic information on age, marital status, educational level, employment status and monthly income were collected. The medical characteristics included time since diagnosis, cancer stage, treatment modalities and comorbidities were also collected. Serum albumin, serum hemoglobin and neutrophils count after receiving at least one course of treatment were retrieved as secondary data. Hypoalbuminemia, low serum hemoglobin and low neutrophils count were categorised using the cut-off points of below $35 \mathrm{~g} / \mathrm{L}, 12.00 \mathrm{~g} / \mathrm{dL}$ and $2.00 \times 10^{9} / \mathrm{L}$, respectively.

\section{Statistical analysis}

All statistical analyses were performed using Statistical Software Package (IBM SPSS statistics version 22.0), with the statistical significance set at $p<0.05$ (two-sided). Patients' characteristics were described as means \pm standard deviations for continuous variables or counts with percentages for categorical variables. The differences in total mean HEl-2015 score were compared according to patients' characteristics, using analysis of variance (ANOVA) for age and month since diagnosis or chi-square test for categorical variables. Associations of HEl-2015 score with overweight, levels of albumin, hemoglobin and neutrophils count were analyzed using binary logistic regression, controlling for demographic and medical characteristics (Model 1). Additional adjustments of moderate-to-vigorous physical activity and/or BMI were performed in Model 2, to examine any changes of observed association in the first model.

\section{Results}

Of 227 breast cancer patients received treatments in hospital, 48 patients were excluded as result of unwillingness to participate $(n=41)$, unable to understand the questions being addressed $(n=1)$, engaged with medical procedures $(n=1)$ and feeling unwell upon recruitment $(n=5)$. A total of 163 patients with completed data was included in the final analysis. Table 2 illustrated the patients' characteristics by quartiles of total HEl-2015 scores. Mean age of the patients was 50.34 years old, with approximately $40 \%$ of them had tertiary education. In this study, the mean duration of treatment was 8.67 months with chemotherapy (57.7\%) as the most common modality received by the patients, followed by a combination of chemotherapy and radiotherapy (37.4\%). Physical inactivity was prevalent with 
approximately two-third of the patients had low level of physical activity, which was accompanied with more than $50 \%$ of the patients were overweight. There was no significant difference between the HEI2015 quartiles with respect to demographic factors of age and education level. The HEl-2015 score was poorer with longer duration of diagnosis, in which post-hoc test revealed that quartile 1 had significantly longer duration of diagnosis than quartile 4. Lower HEI-2015 score was associated with the presence of comorbidity and overweight. None of the subjects was receiving concurrent treatment, while approximately half of them were receiving chemotherapy alone. The overall mean (SD) of HEI score was 63.86 (8.75). A total of $89.6 \%$ subjects $(n=146)$ need dietary improvement, while only $4.3 \%$ subjects $(n=7)$ adhered to dietary guidelines at overall. In term of HEl components (Figure 1), the median scores for adequacy components of Total Fruit, Whole Fruit, Total Vegetables, Greens and Beans, Total Protein Food, Seafood and Plant Proteins, as well as moderation components of Sodium, Added Sugars and Saturated Fats achieved the maximum scores of $100 \%$. More than half of the subjects met the recommendation for above components. On the other hand, Whole Grains, Dairy, Fatty Acids and Refined Grains scored less than $25 \%$ of the maximum score, with only $20 \%$ of patients comply with the recommended intake of these four components.

As shown in Table 3, patients in quartile 4 were less likely to be overweight as compared to patients in quartile 1 after adjusting for demographic and medical characteristics [Model 1: OR=0.36; $95 \% \mathrm{Cl}=0.13,0.99]$. An additional variable of moderate-to-vigorous physical activity did not substantially change this significant association. In model 1, higher diet quality was associated with lower odds of low serum hemoglobin in quartile 3 . The significance value was reduced after adjusting for moderate-tovigorous physical activity and overweight [Model 2: $\mathrm{OR}=0.35 ; 95 \% \mathrm{Cl}=0.13,0.92$ ]. In quartile 4 , the better diet quality was significantly showed with improved serum hemoglobin only after adjusting for behavioural factors [Model 2: OR=0.36; $95 \% \mathrm{Cl}=0.13,0.98$ ].

\section{Discussion}

This study investigated the associations of diet quality with overweight and treatment-related side effects among breast cancer patients undergoing treatment, with most of them underwent chemotherapy alone with a mean duration of therapy was less than 9 months. In this study, the consumptions of whole grains, dairy and fatty acids were low. This finding was consistent with earlier studies ${ }^{23,24}$. Avoidance and abstinence of certain foods are common among cancer patients, owing to belief that animal products is the cause of cancer development ${ }^{19,20}$ or treatment-related side effects that alter the taste perception ${ }^{25}$. On the other hand, dairy products are not the habitual intakes of Malaysian ${ }^{33}$, which may further attribute to the low consumption of dairy products among the patients.

Despite dairy products are good sources for protein, multivitamins and minerals which are essential for bodily function, breast cancer patients may limit their dairy intake attributed to the concern about the safety of growth hormone usage in animal products ${ }^{20}$. World Cancer Research Fund reviewed that the strength and direction of the effect of dairy products on cancer risk is varied across the cancer sites, 
including of prostate, breast and colorectal cancers ${ }^{34,35}$. More studies are needed to confirm the impact of dairy products especially on breast cancer. Contrary to popular belief, the intake of whole grain products that commonly known as healthy food was reduced in breast cancer patients. During treatment, cancer patients experience various side effects which could interfere with the ability of consuming a balanced diet $25,36,37$, in spite of the high awareness of practising healthy eating. The compliance towards refined grain guideline could be deduced from the assessment of whole grains, where both of these components yield identical interpretation. As compared to whole grains, breast cancer patients prefer refined grains products with softer texture such as white rice, white bread or any food made with white flour.

With regards to fatty acid component, the low score is originated from inadequate consumption of monoand poly-unsaturated fatty acids. The individual component of saturated fat reflected that patients limited the food that are high in saturated fat, however, they did not adhere to the guidelines for unsaturated fatty acids. Although there is limited information regarding the fatty acid ratio, a consistent finding of the good compliance with saturated fatty acids intake was documented ${ }^{23,24}$. Polyunsaturated fatty acids from marine sources are suggested to ameliorate treatment-related side effects and improve treatment efficacy via antineoplastic effect ${ }^{38}$. In addition to saturated fatty acid, it is essential to assess the patients' intake of unsaturated fatty acids particularly during treatment. The biggest barrier to adherence to healthy fats recommendation could be high food prices ${ }^{39}$, for instance, margarines with low saturated fat are more expensive than butter that high in saturated fat. This finding may suggest an enhanced accessibility to healthy food by offering affordable price or lower rate than less healthier options. Furthermore, Malaysians hardly consume raw vegetables or fruits accompanied with oil-based dressings, which is not their ordinary food habit. This elucidates that an unhealthy eating habit in general could persist over cancer diagnosis, which may influence patients' prognosis especially during the critical treatment period.

Current finding demonstrated that a better diet quality has protective effect against overweight among breast cancer patients, as supported by Custódio et al. (2016) ${ }^{24}$. Similar relationship between diet quality and obesity has been widely reported by public health studies ${ }^{40-42}$. On the other hand, the finding of the relationship between diet quality and cancer risk or mortality was not consistent ${ }^{13,43}$. As cancer is a multisite disease with different treatment, the relationship of diet with cancer prognosis should be studied according to various cancer types, whereby the development of cancer site-specific diet quality tools is deemed necessary. As previous studies indicated the negative impact of obesity on quality of life ${ }^{44}$ and survival ${ }^{4,5}$ of cancer patients, being overweight is not a desirable outcome for cancer patients who are usually considered to be malnourished. Adopting a healthy eating habit should be a bedrock of lifestyle strategy to achieve a normal weight range as well as to maintain an overall good health. Drenowatz et al. (2014) highlighted that high consumption of protein, sodium and empty calories were the contributors of overweight or obesity among healthy adults ${ }^{41}$, indicating the necessity of achieving an adequate, balanced, moderate and varied diet. 
Iron status of cancer patients should be closely monitored as the incidence of anemia could compromise greatly their quality of life ${ }^{45}$. Current finding showed that higher quality diet was associated with reduced risk of low serum hemoglobin only after adjusting for physical activity and BMI (Model 2), suggesting that diet quality might indirectly improve anemia by reducing excess body weight. It is noteworthy that the low serum hemoglobin could be owing to multiple nutrient deficiencies ${ }^{46,47}$ rather than a single nutrient deficiency. Vegetables and soy food that rich in iron or components enhancing iron absorption were found to associate positively with serum hemoglobin among middle-aged adults and elderly population 47,48 . Vitamin $C$, in addition to iron, was found to be essential to prevent iron deficiency for obese patients who undergone bariatric surgery ${ }^{49}$. Vegetables and soy groups are mainly included in Healthy Eating Index assessment, elucidating the necessity of consuming these components in line with the dietary guidelines. Likewise, Mardas et al. (2017) showed that a whole food rather than a single nutrient was related with chemotherapy-induced gastrointestinal symptoms ${ }^{50}$. Iron rich food or dietary enhancers of iron absorption could be somehow beneficial to anemic cancer patients, despite the low hemoglobin level is generally attributable to cancer or treatment-related factors. With regards to the incidence of anemia, Thomson et al. (2011) recommended to keep body weight within a healthy range $\mathrm{e}^{46}$. Inadequate diet intake could predispose patients to iron deficiency while obese women are likely to experience interrupted iron absorption ${ }^{46,48}$. Despite the potential linkage of diet quality with serum hemoglobin, this marker should be interpreted carefully as it may be affected by cancer prognosis related inflammation ${ }^{51}$.

In view of the potential inter-relationship between health indicators, investigation on the mediating effect of body mass index in respect to the association between diet quality and low serum hemoglobin are warranted. A change in body weight is explained by the fundamental principle of energy balance, where an imbalance between energy content of food consumed and energy output of basal energy expenditure and daily activity performance occurs. To our knowledge, physical inactivity is an established risk factor for overweight. In spite of that, current study failed to show the pivotal role of physical activity in the association between diet quality and overweight among breast cancer patients during treatment. The increased strength of the relationship between diet quality and serum hemoglobin after controlling the behavioral factors may only be due to overweight.

Noting of the non-prospective study design, this finding does not preclude the notion of substantial impact of poor diet quality on the burden of multiple health outcomes. Despite demographic factors are considered as the risk factors of unhealthy diet in the causation pathway for non-communicable diseases ${ }^{10}$, none of the variables was shown to associate with diet quality in current study. This could be explained by the varying extent of demographic influences on diet behaviour across the population groups.

Present study yields new insights into how a quality diet was linked to the clinical outcomes of patients with breast cancer, with the evaluation of the degree of conformance in consumption of various food groups with dietary guidelines. Achieving a healthy weight range has been set as a primary goal to 
prevent nutrient deficiencies and to ensure an optimal health for cancer population ${ }^{52}$. The assessment of diet quality in breast cancer patients, particularly those receiving treatment, therefore, provides valuable information for the identification of patients' nutritional status during this critical phase. Moreover, the association between diet quality and treatment-related side effects was performed using multivariate analysis, together with the adjustment of multiple confounders. This minimizes the bias that resulted from the factors that are out of study interest.

The limitations of this study included cross sectional design which determined both exposure and outcome simultaneously, whereby, the causal effect of poor diet towards treatment-related side effects could not be assured. The limited sample size of this study may result in the issue of data sparsity, which may not be representative of the overall findings. In light of the need for increased power of study and strength of evidence, a larger sample size should be considered in the future studies. The significant associations between diet quality and overweight or low haemoglobin should be interpreted with cautious as the confidence interval for odds ratio were closed to null value of 1 indicates weak implication of results. As the patient-reported outcomes rely on cancer types and treatment factors, these findings may not be generalized to other cancer groups or trajectory phases. For instance, the study population were mainly characterized by non-terminal stage, in which the study finding could not be extrapolated to breast cancer patients under hospice care with poor prognosis. The measurement periods of blood serum or body weight were inconsistent across phases of cancer treatment, which does not allow parallel comparison with 1 month of diet history. This implies that the dietary assessment over the past one month is insufficient to reflect the overall dietary pattern during treatment and the assessment period should be extended considering the data reliability without compensating patients' burden. Besides, short assessment period of laboratory measures and body weight may also be insufficient to reflect the nutritional status of patients throughout the treatment, which may undermine the study findings.

Despite Healthy Eating Index was found to be a well-established tool to predict obesity ${ }^{53}$, it does not consider the key principle of balance, as referring that each food groups should be taken in right amount or proportion. In regard to this limitation, an underestimation of over-eating might be existed. For instance, though protein is important for muscle building, over-consumption of proteins can contribute significant calorie which could lead to overweight. Lack of nutrition information, particularly for saturated, mono- and polyunsaturated fatty acids, may underscore the component of fatty acid in overall diet quality evaluation. However, additional food databases with higher availability of food items or nutrients were utilized in order to minimize such bias.

\section{Conclusions}

In conclusion, breast cancer patients reported that their overall diet quality during treatments need improvement. A better diet quality was statistically associated with lower likelihood of overweight, however, the strength of such association was weak. On the other hand, non-significant associations were shown between diet quality and altered laboratory values. Findings from this study may serve as fundamental data regarding the impact of diet quality on overweight, which enable healthcare 
practitioners to implement effective strategies in the improvement of patients' nutritional status and cancer care. More investigations are deemed necessary to identify the exact mechanism of how diet links to overweight among breast cancer patients.

\section{List Of Abbreviations}

\begin{tabular}{ll} 
MREC & Medical Research and Ethics Committee \\
\hline IPAQ-SF & International Physical Activity Questionnaire-short form \\
\hline FFQ & food frequencies questionnaires \\
HEI & Healthy eating index \\
BMI & Body mass index
\end{tabular}

\section{Declarations}

\section{Ethics approval and consent to participate}

Ethics approval was provided by Medical Research and Ethics Committee (MREC), Ministry of Health Malaysia and Ethics Committee for Research Involving Human Subject Universiti Putra Malaysia. Patients' written informed consents were obtained prior to study enrolment.

\section{Consent for publication}

Not applicable.

\section{Availability of data and materials}

The datasets used and/or analysed during the current study are available from the corresponding author on reasonable request.

\section{Competing interests}

The authors declare no conflict of interest regarding the publication of this articles.

\section{Funding}

This work was funded by the Universiti Putra Malaysia (grant number GP-IPS 9507400). This funding body has no role in the design of the study and collection, analysis, and interpretation of data and in writing the manuscript.

\section{Authors' contributions}


CYM designed the study. KNLS collected, computed, analysed and interpreted the data. CYM and ZMS supervised the entire process. KNLS prepared the manuscript while CYM and ZMS reviewed and edited the manuscript. All authors approved the final manuscript.

\section{Acknowledgements}

Special thanks are dedicated to all patients and staffs of National Cancer Institute at Putrajaya, for the enthusiasm in data collection. The authors thank Dr Subashini Elangkovan from the Clinical Research Centre; Norshariza Jamhuri, Betti Sharina Mohd Haniff, Siti Nuraini Mohd Samwil, and Ng Wai Han from the Department of Dietetics, for assistance along the data collection.

\section{References}

1. Hébuterne X, Lemarié E, Michallet M, De Montreuil CB, Schneider SM, Goldwasser F. Prevalence of malnutrition and current use of nutrition support in patients with cancer. J Parenter Enter Nutr. 2014;38(2):196-204.

2. Pressoir M, Desné S, Berchery D, Rossignol G, Poiree B, Meslier M, Traversier S, Vittot M, Simon M, Gekiere JP et al. Prevalence, risk factors and clinical implications of malnutrition in french comprehensive cancer centres. Br J Cancer. 2010;102(6):966-971.

3. van den Berg MMGA, Winkels RM, de Kruif JT, van Laarhoven HW, Visser M, de Vries JHM, de Vries YC, Kampman E. Weight change during chemotherapy in breast cancer patients: a meta-analysis. BMC Cancer. 2017;17(1):259.

4. Jiralerspong S, Goodwin PJ. Obesity and breast cancer prognosis: Evidence, challenges, and opportunities. J Clin Oncol. 2016;34(35):4203-4216.

5. Picon-ruiz M, Morata-tarifa C, Valle-goffin JJ, Friedman ER, Slingerland JM. Obesity and Adverse Breast Cancer Risk and Outcome: Mechanistic Insights and Strategies for Intervention. A Cancer Journals Clin. 2017;67(5).

6. Tachi T, Teramachi H, Tanaka K, Asano S, Osawa T, Kawashima A, Yasuda M, Mizui T, Nakada T, Noguchi $Y$ et al. The impact of outpatient chemotherapy-related adverse events on the quality of life of breast cancer patients. PLoS One. 2015;10(4):1-15.

7. Bower JE. Cancer-related fatigue: Mechanisms,risk factors and treatments. Nat Rev Clin Oncol. 2014;11(10):597-609.

8. Lyman GH, Michels SL, Reynolds MW, Barron R, Tomic KS, Yu J. Risk of mortality in patients with cancer who experience febrile neutropenia. Cancer. 2010;116(23):5555-5563.

9. Kane HL, Halpern MT, Squiers LB, Treiman KA, McCormack LA. Implementing and Evaluating Shared Decision Making in Oncology Practice. CA Cancer J Clin. 2014;64(6):377-388.

10. Dans A, Ng N, Varghese C, Tai ES, Firestone R, Bonita R. The rise of chronic non-communicable diseases in southeast Asia: Time for action. Lancet. 2011;377(9766):680-689. 
11. Gal R, Monninkhof EM, Peeters PHM, van Gils CH, van den Bongard DHJG, Wendel-Vos GCW, Zuithoff NPA, Verkooijen HM, May AM. Physical activity levels of women with breast cancer during and after treatment, a comparison with the Dutch female population. Acta Oncol. 2019;58(5):673681.

12. Wirt A, Collins CE. Diet quality - What is it and does it matter? Public Health Nutr. 2009;12(12):24732492.

13. Du M, Liu SH, Mitchell C, Fung TT. Associations between diet quality scores and risk of postmenopausal estrogen receptor-negative breast cancer: A systematic review. J Nutr. 2018;148(1):100-108.

14. George SM, Neuhouser ML, Mayne ST, Irwin ML, Albanes D, Gail MH, Alfano CM, Bernstein L, McTiernan A, Reedy $\mathrm{J}$ et al. Postdiagnosis diet quality is inversely related to a biomarker of inflammation among breast cancer survivors. Cancer Epidemiol Biomarkers Prev. 2010;19(9):22202228.

15. George SM, Ballard-Barbash R, Shikany JM, Caan BJ, Freudenheim JL, Kroenke CH, Vitolins MZ, Beresford SA, Neuhouser ML. Better Postdiagnosis Diet Quality Is Associated with Reduced Risk of Death among Postmenopausal Women with Invasive Breast Cancer in the Women's Health Initiative. Cancer Epidemiol Biomarkers Prev. 2014;23(4):575-583.

16. Terranova CO, Protani MM, Reeves MM. Overall Dietary Intake and Prognosis after Breast Cancer: A Systematic Review. Nutr Cancer. 2018;70(2):153-163.

17. Jacobs DR, Gross MD, Tapsell LC. Food synergy: an operational concept for understanding nutrition. Am J Clin Nutr. 2009;89(5):1543-1548.

18. Wu G. Dietary protein intake and human health. Food Funct. 2016;7(3):1251-1265.

19. Beeken RJ, Williams K, Wardle J, Croker H. "What about diet?" A qualitative study of cancer survivors' views on diet and cancer and their sources of information. Eur J Cancer Care (Engl). 2016;25(5):774783.

20. Thomson AK, Heyworth JS, Girschik J, Slevin T, Saunders C, Fritschi L. Beliefs and perceptions about the causes of breast cancer: a case-control study. BMC Res Notes. 2014;7:558.

21. Shaharudin SH, Sulaiman S, Shahril MR, Emran NA, Akmal SN. Dietary changes among breast cancer patients in Malaysia. Cancer Nurs. 2013;36(2):131-8.

22. Yong HY, Shariff ZM, Kandiah M, Yong HW, Saibul N, Sariman S, Hashim Z. Diet and physical activity in relation to weight change among breast cancer patients. Asian Pac J Cancer Prev. 2014;15(1):3944.

23. Ceccatto V, Faria Di Pietro P, Nogueira Previdelli A, Kunradi Vieira FG, Cesa Schiavon C, Engel R, Lizane Cardoso A, Altenburg de Assis MA, Gilberto Crippa C, Gonzalez Chica DA. Brazilian healthy eating index revised (BHEI-R) of women before and during adjuvant treatment for breast cancer. Nutr Hosp. 2014;30(5):1101-1109.

24. Custódio IDD, Marinho EDC, Gontijo CA, Pereira TSS, Paiva CE, Maia YC de P. Impact of chemotherapy on diet and nutritional status of women with breast cancer: A prospective study. PLoS 
One. 2016;11(6):1-21.

25. Marinho E da C, Custódio IDD, Ferreira IB, Crispim CA, Paiva CE, Maia YC de P. Impact of chemotherapy on perceptions related to food intake in women with breast cancer: A prospective study. PLoS One. 2017;12(11):1-14.

26. Jochems SHJ, Van Osch FHM, Bryan RT, Wesselius A, van Schooten FJ, Cheng KK, Zeegers MP. Impact of dietary patterns and the main food groups on mortality and recurrence in cancer survivors: A systematic review of current epidemiological literature. BMJ Open. 2017;8(2):1-12.

27. Leenders M, Sluijs I, Ros MM, Boshuizen HC, Siersema PD, Ferrari P, Weikert C, Tjønneland A, Oisen A, Boutron-Ruault $\mathrm{M}$ et al. Fruit and vegetable consumption and mortality. Am J Epidemiol. 2013;178(4):590-602.

28. World Health Organization. Regional Office for the Western Pacific. The Asia-Pacific Perspective: Redefining Obesity and Its Treatment. Sydney: Health Communications Australia. 2000. Avialable from http://www.wpro.who.int/nutrition/documents/docs/Redefiningobesity.pdf

29. Institute for Public Health. National Health and Morbidity Survey 2014: Malaysian Adult Nutrition Survey Volume I: Methodology and General Findings. 2014. Available from http://iku.moh.gov.my/images/IKU/Document/REPORT/NHMS2014-MANS-VOLUME-1MethodologyandGeneralFind.pdf

30. National Cancer Institute; National Institutes of Health. Overview and Background of The Healthy Eating Index. [Internet]. 2018. Available from https://epi.grants.cancer.gov/hei/. Cited 19 July 2018.

31. Fokeena WB, Jamaluddin R, Khaza'ai H. Development and Assessment of the Reliability and Validity of a Diet Quality Index in a Sample of Malaysian University Students. J Food Nutr Res. 2016;4(4):251-257.

32. IPAQ (2005). Guidelines for data processing and analysis of the International Physical Activity Questionnaire (IPAQ) - short and long forms. 2005. Available from https://sites.google.com/site/theipaq/scoring-protocol

33. Shahril MR, Sulaiman S, Shaharudin SH, Akmal SN. Healthy eating index and breast cancer risk among Malaysian women. Eur J Cancer Prev. 2013;22(4):342-347.

34. World Cancer Research Fund, American Institute for Cancer Research. Diet, Nutrition, Physical Activity and Cancer: A Global Perspective. Meat, Fish and Dairy Products and the Risk of Cancer. 2018. Available from https://www.wcrf.org/sites/default/files/Meat-Fish-and-Dairy-products.pdf.

35. World Cancer Research Fund/American Institute for Cancer Research. Diet, Nutrition, Physical Activity and Cancer: A Global Perspective. [Internet]. 2018. Available from https://www.wcrf.org/dietandcancer. Cited 31 July 2019.

36. Boltong A, Aranda S, Keast R, Wynne R, Francis PA, Chirgwin J, Gough K. A prospective cohort study of the effects of adjuvant breast cancer chemotherapy on taste function, food liking, appetite and associated nutritional outcomes. PLoS One. 2014;9(7):1-9.

37. de Vries YC, van den Berg MMGA, de Vries JHM, Boesveldt S, DE Kruif JTCM, Buist N, Haringhuizen A, Los M, Sommeijer DW, Timmer-Bonte JHN et al. Differences in dietary intake during chemotherapy 
in breast cancer patients compared to women without cancer. Support Care Cancer. 2017;25(8):25812591.

38. D'Eliseo D, Velotti F. Omega-3 Fatty Acids and Cancer Cell Cytotoxicity: Implications for MultiTargeted Cancer Therapy. J Clin Med. 2016;5(2):15.

39. Rao M, Afshin A, Singh G, Mozaffarian D. Do healthier foods and diet patterns cost more than less healthy options? A systematic review and meta-analysis. BMJ Open. 2013;3(12).

40. Livingstone KM, McNaughton SA. Diet quality is associated with obesity and hypertension in Australian adults: a cross sectional study. BMC Public Health. 2016;16(1):1-10.

41. Drenowatz C, Shook RP, Hand GA, Hébert JR, Blair SN. The independent association between diet quality and body composition. Sci Rep. 2014;4:1-6.

42. Drewnowski A, Aggarwal A, Tang W, Hurvitz PM, Scully J, Stewart O, Moudon AV. Obesity, diet quality, physical activity, and the built environment: the need for behavioral pathways. BMC Public Health. 2016;16(1):1-12.

43. Potter J, Brown L, Williams RL, Byles J, Collins CE. Diet quality and cancer outcomes in adults: A systematic review of epidemiological studies. Int J Mol Sci. 2016;17(7):1-30.

44. Fang P, Tan KS, Troxel AB, Rengan R, Freedman G, Lin LL. High body mass index is associated with worse quality of life in breast cancer patients receiving radiotherapy. Breast Cancer Res Treat. 2013;141(1):125-133.

45. Kanuri G, Sawhney R, Varghese J, Britto M, Shet A. Iron deficiency anemia coexists with cancer related anemia and adversely impacts quality of life. PLoS One. 2016;11(9):1-11.

46. Thomson CA, Stanaway J, Neuhouser ML, Snetselaar LG, Stefanick ML, Arendell L, Chen Z. Nutrient Intake and Anemia Risk in the WHI Observational Study. J Am Diet Assoc. 2011;111(4):532-541.

47. Zhang Q, Qin G, Liu Z, Li Z, Li J, Varma DS, Wan Q, Zhao J, Min X, Han X et al. Dietary balance index07 and the risk of anemia in middle aged and elderly people in southwest China: A cross sectional study. Nutrients. 2018;10(2):1-12.

48. Song P, Li L, Man Q, Wang C, Meng L, Zhang J. Case-control study of anaemia among middle-aged and elderly women in three rural areas of China. BMJ Open. 2014;4(8).

49. Mischler RA, Armah SM, Wright BN, Mattar SG, Rosen AD, Gletsu-Miller N. Influence of diet and supplements on iron status after gastric bypass surgery. Surg Obes Relat Dis. 2016;12(3):651-658.

50. Mardas M, Madry R, Stelmach-Mardas M. Link between diet and chemotherapy related gastrointestinal side effects. Wspolczesna Onkol. 2017;21(2):162-167.

51. Fankhauser CD, Sander S, Roth L, Goss O, Eberli D, Sulser T, Seifert B, Beyer J, Hermanns T. Systemic inflammatory markers have independent prognostic value in patients with metastatic testicular germ cell tumours undergoing first-line chemotherapy. Br J Cancer. 2018;118(6):825-830.

52. Rock CL, Doyle C, Demark-Wahnefried W, Meyerhardt J, Courneya KS, Schwartz AL, Bandera EV, Hamilton KK, Grant B, McCullough M et al. Nutrition and Physical Activity Guidelines for Cancer Survivors. CA Cancer J Clin. 2012;00:000-000. 
53. Asghari G, Mirmiran P, Yuzbashian E, Azizi F. A systematic review of diet quality indices in relation to obesity. Br J Nutr. 2017;117(8):1055-1065.

\section{Tables}

Table 1: Standard scores of HEI-2015 components

\begin{tabular}{lccc}
\hline HEI-2015 components & Maximum scores & Standard for maximum score & Standard for minimum score \\
\hline Adequacy & & & \\
$\quad$ Total fruits & 5 & $\geq 0.8 \mathrm{cup}$ & No Fruits \\
\hline Whole fruits & 5 & $\geq 1.1 \mathrm{cup}$ & No Whole Fruits \\
\hline Total vegetables & 5 & $\geq 0.2 \mathrm{cup}$ & No Vegetables \\
\hline Greens and beans & 5 & $\geq 1.5 \mathrm{oz}$ & No Greens and Beans \\
\hline Whole grains & 10 & $\geq 1.3 \mathrm{cup}$ & No Whole Grains \\
\hline Dairy & 10 & $\geq 2.5 \mathrm{oz}$ & No Dairy \\
\hline Total Protein Food & 5 & $\geq 0.8 \mathrm{cup}$ & No Seafood and Protein food \\
\hline Seafood and Plant Proteins & 5 & (PUFAs + MUFAs)/SFAs $\geq 2.5$ & (PUFAs + MUFAs) $/$ SFAs $\leq 1.2$ \\
\hline Fatty Acids & 10 & $\leq 1.8 \mathrm{oz}$ & $\geq 4.3$ oz \\
\hline Moderation & & $\leq 1.1 \mathrm{~g}$ & $\geq 2.0 \mathrm{~g}$ \\
\hline Refined Grains & 10 & $\leq 6.5 \%$ of energy & $\geq 26 \%$ of energy \\
\hline Sodium & 10 & $\leq 8 \%$ of energy & $\geq 16 \%$ of energy \\
\hline Added sugars & 10 & 10 &
\end{tabular}

Table 2: Distribution of Patients' characteristics as stratified by Diet Quality Quartiles 


\begin{tabular}{|c|c|c|c|c|c|}
\hline \multirow[t]{2}{*}{ Parameters } & \multicolumn{4}{|c|}{ Quartiles of HEI-2015 score } & \multirow[t]{2}{*}{$p$} \\
\hline & $\begin{array}{c}1 \\
(41 \text { to }<58.7)\end{array}$ & $\begin{array}{c}2 \\
(58.7 \text { to }<62.5)\end{array}$ & $\begin{array}{c}3 \\
(62.5 \text { to }<69.5)\end{array}$ & $\begin{array}{c}4 \\
\text { (69.5 to 89) }\end{array}$ & \\
\hline Number of patients & 41 & 41 & 41 & 40 & \\
\hline Age (years), mean \pm SD & $50.95 \pm 11.40$ & $48.83 \pm 10.28$ & $51.41 \pm 10.09$ & $50.15 \pm 9.13$ & 0.684 \\
\hline Educational level & & & & & 0.567 \\
\hline Secondary level and below & $25(61.0)$ & $27(65.9)$ & $21(51.2)$ & $25(62.5)$ & \\
\hline Higher education & $16(39.0)$ & $14(34.1)$ & $20(48.8)$ & $15(37.5)$ & \\
\hline Month since diagnosis, mean \pm SD & $11.01 \pm 6.69 *$ & $8.28 \pm 5.96$ & $8.00 \pm 5.63$ & $7.31 \pm 4.92 *$ & 0.027 \\
\hline Cancer stage & & & & & 0.380 \\
\hline $0 / \mathrm{I} / \mathrm{II}$ & $12(29.3)$ & $16(39.0)$ & $16(39.0)$ & $16(40.0)$ & \\
\hline III & $19(46.3)$ & $15(36.6)$ & $18(43.9)$ & $10(25.0)$ & \\
\hline IV & $10(24.4)$ & $10(14.4)$ & $7(17.1)$ & $14(35.0)$ & \\
\hline Chemotherapy (ongoing) & & & & & 0.164 \\
\hline Yes & $21(51.2)$ & $25(61.0)$ & $29(70.7)$ & $29(72.5)$ & \\
\hline No & $20(48.8)$ & $16(39.0)$ & $12(29.3)$ & $11(27.5)$ & \\
\hline Radiotherapy (ongoing) & & & & & 0.061 \\
\hline Yes & $13(31.7)$ & $11(26.8)$ & $5(12.2)$ & $5(12.5)$ & \\
\hline No & $28(68.3)$ & $30(73.2)$ & $36(87.8)$ & $35(87.5)$ & \\
\hline Hormonal therapy (ongoing) & & & & & 0.316 \\
\hline Yes & $13(31.7)$ & $7(17.1)$ & $7(17.1)$ & $8(20.0)$ & \\
\hline No & $28(68.3)$ & $34(82.9)$ & $34(82.9)$ & $32(80.0)$ & \\
\hline Targeted therapy (ongoing) & & & & & 0.904 \\
\hline Yes & $7(17.1)$ & $5(12.2)$ & $6(14.6)$ & $7(17.5)$ & \\
\hline No & $34(82.9)$ & $36(87.8)$ & $35(85.4)$ & $33(82.5)$ & \\
\hline Combined/single treatment modalities & & & & & $0.062 \dagger$ \\
\hline CT alone & $19(46.3)$ & $24(58.5)$ & $27(65.9)$ & $24(60.0)$ & \\
\hline RT alone & $3(7.3)$ & $5(12.2)$ & $0(0.0)$ & $0(0.0)$ & \\
\hline $\mathrm{CT}+\mathrm{RT}$ & $19(46.3)$ & $12(29.3)$ & $14(34.1)$ & $16(40.0)$ & \\
\hline Comorbidity & & & & & 0.021 \\
\hline Yes & $24(58.5)$ & $13(31.7)$ & $22(53.7)$ & $13(32.5)$ & \\
\hline No & $17(41.5)$ & $28(68.3)$ & $19(46.3)$ & $27(67.5)$ & \\
\hline Meets moderate/vigorous PA & & & & & 0.779 \\
\hline Yes & $12(29.3)$ & $14(34.1)$ & $15(36.6)$ & $16(40.0)$ & \\
\hline No & $29(70.7)$ & 27 (65.9) & $26(63.4)$ & $24(60.0)$ & \\
\hline Overweight & & & & & 0.021 \\
\hline Yes & $28(68.3)$ & $19(46.3)$ & $23(56.1)$ & $14(35.0)$ & \\
\hline No & $13(31.7)$ & $22(53.7)$ & $18(43.9)$ & $26(65.0)$ & \\
\hline Hypoalbuminemia & & & & & 0.691 \\
\hline Yes & $3(7.3)$ & $3(7.3)$ & $4(9.8)$ & $1(9.1)$ & \\
\hline No & $38(92.7)$ & $38(92.7)$ & $37(90.2)$ & $39(97.5)$ & \\
\hline Low haemoglobin & & & & & $0.186 \dagger$ \\
\hline Yes & $28(68.3)$ & $25(62.5)$ & $19(46.3)$ & $21(52.5)$ & \\
\hline No & $13(31.7)$ & $15(37.5)$ & $22(53.7)$ & $19(47.5)$ & \\
\hline Low neutrophils count & & & & & 0.700 \\
\hline Yes & $3(7.3)$ & $5(12.2)$ & $6(14.6)$ & $6(15.0)$ & \\
\hline No & $38(92.7)$ & $36(87.8)$ & $35(85.4)$ & $34(85.0)$ & \\
\hline
\end{tabular}

Data were presented as mean \pm SD or number (frequency)

Figure in bold indicate statistical significance based on ANOVA and chi-square test

*Post-hoc test revealed that Q1 had significantly higher score than Q4 $(\mathrm{p}=0.025)$

†Statistical significant based on fisher's exact test 
Table 3: Multivariate associations of total HEI-2015 score with overweight status and treatment-related side effects

\begin{tabular}{|c|c|c|c|c|c|c|}
\hline & \multicolumn{6}{|c|}{ HEI-2015 score (ref: Quartile $1=41$ to $<58.7$ ) } \\
\hline & \multicolumn{2}{|c|}{$\begin{array}{c}2 \\
(58.7 \text { to }<62.5)\end{array}$} & \multicolumn{2}{|c|}{$\begin{array}{c}3 \\
(62.5 \text { to }<69.5)\end{array}$} & \multicolumn{2}{|l|}{$\begin{array}{c}4 \\
(69.5 \text { to 89) } \\
\end{array}$} \\
\hline & OR (95\% CI) & $p$ & OR $(95 \% \mathrm{CI})$ & $\mathrm{p}$ & OR $(95 \% \mathrm{CI})$ & $\mathrm{p}$ \\
\hline \multicolumn{7}{|c|}{ Overweight $^{\mathrm{a}}$} \\
\hline Model 1 & $0.58(0.22,1.54)$ & 0.270 & $0.70(0.26,1.83)$ & 0.463 & $0.36(0.13,0.99)$ & 0.047 \\
\hline Model 2 & $0.58(0.22,1.54)$ & 0.270 & $0.70(0.26,1.83)$ & 0.462 & $0.36(0.13,0.99)$ & 0.047 \\
\hline \multicolumn{7}{|c|}{ Hypoalbuminemia $^{b}$} \\
\hline Model 1 & $0.94(0.13,6.74)$ & 0.951 & $1.21(0.20,7.18)$ & 0.832 & $0.17(0.01,2.15)$ & 0.170 \\
\hline Model 2 & $0.87(0.11,6.73)$ & 0.898 & $1.20(0.19,7.52)$ & 0.847 & $0.13(0.10,1.96)$ & 0.142 \\
\hline \multicolumn{7}{|c|}{ Low hemoglobin ${ }^{\mathrm{b}}$} \\
\hline Model 1 & $0.65(0.24,1.76)$ & 0.398 & $0.37(0.14,0.95)$ & 0.039 & $0.39(0.14,1.05)$ & 0.062 \\
\hline Model 2 & $0.64(0.24,1.72)$ & 0.374 & $0.35(0.13,0.92)$ & 0.033 & $0.36(0.13,0.98)$ & 0.046 \\
\hline \multicolumn{7}{|c|}{ Low neutrophils count ${ }^{b}$} \\
\hline Model 1 & $1.47(0.26,8.32)$ & 0.662 & $2.85(0.55,14.89)$ & 0.214 & $2.36(0.44,12.63)$ & 0.317 \\
\hline Model 2 & $1.15(0.17,7.65)$ & 0.887 & $2.63(0.45,15.59)$ & 0.286 & $1.88(0.31,11.34)$ & 0.492 \\
\hline
\end{tabular}

$\mathrm{n}=163$ for each model fitted

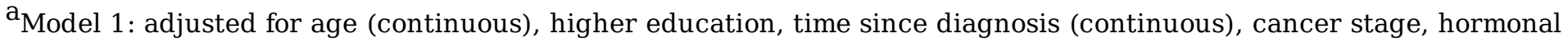
therapy, comorbidity; Model 2: Model 1 + moderate-to-vigorous PA;

$\mathrm{b}$ Model 1: adjusted for age (continuous), higher education, time since diagnosis (continuous), cancer stage, hormonal therapy, comorbidity; Model 2: Model $1+$ moderate-to-vigorous PA + overweight

\section{Figures}


Figure 1: Radar graph of median components score in HEI-2015 and percentage of meeting dietary recommendations

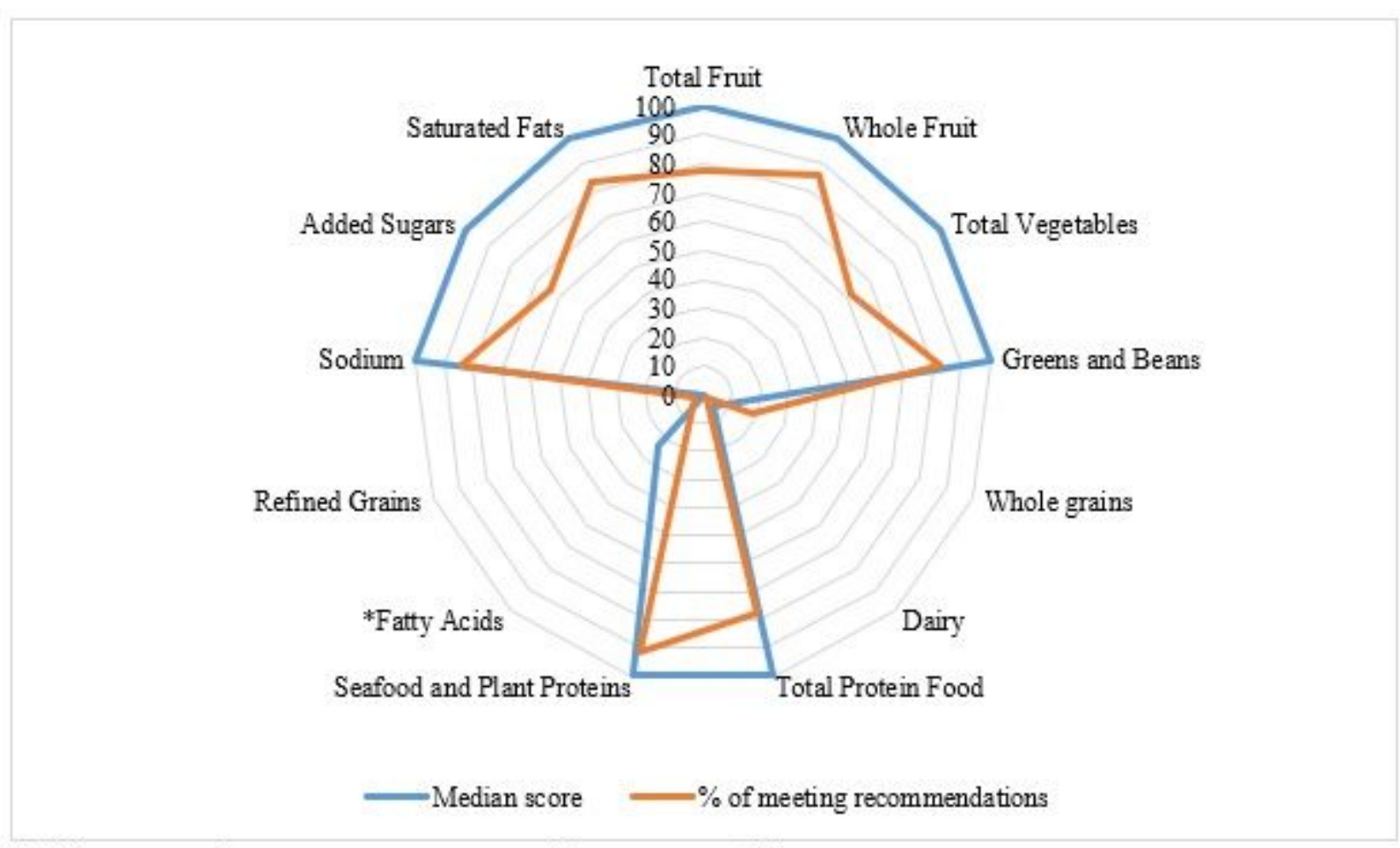

Median scores of component were expressed in percentage (\%)

Higher $\%$ indicate better conformance towards dietary guidelines

*Fatty Acids: ratio of poly- and mono-unsaturated fatty acids to saturated fatty acids

\section{Figure 1}

Radar graph of median components score in HEl-2015 and percentage of meeting dietary recommendations

\section{Supplementary Files}

This is a list of supplementary files associated with this preprint. Click to download.

- Tables.pdf 